\title{
Decision-Making Style in Leaders: Uncovering Cognitive Motivation Using Signature Movement Patterns
}

\author{
Brenda L. Connors ${ }^{1}$, Richard Rende ${ }^{2} \&$ Timothy J. Colton ${ }^{3}$ \\ ${ }^{1}$ CNO Strategic Studies Group, Newport, RI \\ ${ }^{2}$ Social Behavioral Research Applications, Phoenix, AZ \\ ${ }^{3}$ Harvard University, Cambridge, MA \\ Correspondence: Brenda L. Connors, CNO Strategic Studies Group, 686 Cushing Road, Newport, RI \\ 02841-1207, USA. Tel: 1-401-841-6571. E-mail: connorsb@usnwc.edu
}

Received: March 18, 2015

Accepted: April 1, 2015

Online Published: May 29, 2015

doi:10.5539/ijps.v7n2p105

URL: http://dx.doi.org/10.5539/ijps.v7n2p105

\begin{abstract}
Leaders can and do vary substantially in their decision-making styles. One promising approach for capturing such variation is to analyze signature movement patterns that reflect cognitive priorities and motivations manifested during various stages of the decision making process. Prior work has shown that Assertion and Perspective - two broad dimensions of decision-making style derived from application of Movement Pattern Analysis (MPA) - reliably predict individual differences in decision making process as assessed experimentally. In this study, we examine underlying motivational propensities as applied by leaders across stages of the decision-making process to illuminate the most salient indicators of decision-making style, ones that are predictive of variations in decision-making behavior. Motivations across three stages of decision making revealed by MPA - Attention, Intention, and Commitment - were recorded from observational data by trained coders. While individual differences were notable across all three stages, those associated with Intention were most predictive of two indicators of decision-making process recorded during laboratory assessment. The implications of this work for capturing indicators of motivational bases of individual differences in decision-making style in high-level leaders are discussed.
\end{abstract}

Keywords: Movement Pattern Analysis (MPA), decision-making style, decision-making stages, individual differences, leadership

\section{Introduction}

Although leaders undoubtedly share many characteristics, there is increasing recognition that they can vary substantially in a number of stylistic aspects of their actions. Particular attention has been directed toward the notable differences in decision-making style that can be observed when studying a group of leaders (see Connors, Rende, \& Colton, 2013; Connors, Rende, \& Colton, 2014). For example, prior studies have shown notable variation in decision-making style in experienced leaders in general (Carnevale, Inbar, \& Lerner, 2011), leaders across different types of professional background (Laureiro-Martinez et al., 2014), and military officers (Thunholm, 2004).

Recent research has taken a more fine-grained approach to identifying specific processes that drive the observable individual differences in decision-making style of potential interest to the study of leaders. One promising line of work has focused on the different stages of cognitive effort that comprise the decision making process (White et al., 2014). From a methodological perspective, individual differences become prominent when paradigms are used that permit subjects to direct their own decision-making processes (see Barnes, Anderson, Plitt, \& Martin, 2014), rather than adhere to strict constraints in time (e.g., requiring a decision within a specific window of time) and choice (e.g., offering limited choices based on restricted information). For instance, while the manner in which subjects balance speed and accuracy concerns (two considerations that come into play in certain decision-making contexts) is often directly manipulated in the laboratory via instruction, individual differences in spontaneous behavioral tendencies emerge when no such manipulation is offered. The result for the investigator is an ability to discern how different people coordinate these two interacting but separate processing strategies in different ways (Perri, Berchicci, Spinelli, \& Di Russo, 2014). A similar perspective on 
individual differences in decision-making strategies emerges when examining use of prior knowledge during decisions (Hansen, Hillenbrand, \& Ungerleider, 2012), coordinating habitual and goal-directed decision-making (Eppinger, Walter, Heekeren, \& Li, 2013), and employment of multiple memory systems to serve different computational strategies during decision-making (Doll, Shohamy, \& Daw, 2015). Given this prominent attention to utilizing new methods that elicit, rather than inhibit, individual differences (see Connors et al., 2013), it is perhaps not surprising that we see an accumulation of robust evidence supporting the variability of decision-making strategies (e.g., Mishra, 2014; White et al., 2014), including more precision in identifying specific stages in movement cognitive processing that best reveal decision-making style.

Individual differences in components of decision-making process can be reliably elicited in the laboratory, but a challenge is to develop methods that can reliably predict decision-making style, particularly on the part of leaders of interest such as military, business executives, and heads of state. Most methods used to date have focused on self-report, which while valuable carry some limitations. For example, it is not clear the extent to which individuals have the necessary insight into their own decision-making style to serve as the sole or primary source of information about that variable. Furthermore, in some fields, the analyst may want mainly to capture information on decision-making style in leaders who would not be available for self-assessment or direct study, such as heads of state (e.g., Connors, 2006; Connors et al., 2013).

One promising approach that addresses these concerns is to focus on methods that capture signature movement patterns observable in a number of ways and codable by trained experts. These, we submit, reliably predict decision-making process at the individual level. In this paper, we focus on Movement Pattern Analysis (MPA), a theory-driven observational methodology which has been used for decades in business to guide executive recruitment, position selection, and the building of management teams, and has been applied in physical and psychological therapies (see Lamb, 2012; Moore, 2005). Briefly, MPA centers on the recording and coding of a number of "posture-gesture mergers" (PGMs), which are observable behaviors that are considered to correspond to multiple stages of the decision-making process (see Connors et al., 2013; Connors et al., 2014, for more detailed description).

In the MPA framework, PGMs are used to generate two Overall Factors-Assertion and Perspective- that together represent a signature decision-making style. The core idea is that individuals need to balance multiple actions/motivations throughout stages of decision making (see Connors et al., 2013; Connors et al., 2014). Assertion reflects the exertion of tangible energy (physical and mental) in the environment in order to get results and make things happen. Perspective involves shaping the body to position oneself to receive from the environment information to derive insight on how a decision relates to the whole or to other decisions. Differences in how individuals achieve their own balance between Assertion and Perspective capture different decision-making styles. For example, individuals high on Assertion rely upon decision-making motivations that include intensively focusing to probe and gather knowledge, applying pressure to support determination, and pacing in time to reach a decision at just the right moment. In contrast, individuals high on Perspective are considered more strategic and are receptive to a broad scope of ideas and information alternatives - they shape their bodily position to reflect on the decision's relative value or priority and in such a way to gain perspective on how the stages of decision implementation may strategically achieve an overall outcome. Connors et al. (2013) provide examples of signature movements reflecting Assertion and Perspective.

MPA has been shown to yield excellent inter-rater reliability (Connors et al., 2013), a necessity for any observational methodology. Furthermore, recent experimental work has demonstrated that MPA is predictive of decision-making process as elicited in the laboratory (Connors et al., 2013; Connors et al., 2014). A sample of military leaders were shown to vary substantially in the way in which they balanced the need for Assertion and Perspective, based upon their MPA profile. Importantly, these individual differences in decision-making motivation were highly predictive of their recorded decision-making processes, evoked via completion of a battery of hypothetical decision-making scenarios in which subjects could control the amount of information they needed before coming to a decision (Connors et al., 2013). Those who relied more upon Perspective as opposed to Assertion reached for more information before arriving at a decision, and had overall longer reaction times. Further work revealed that the type of patterning that is coded in MPA - the individually-referenced balance between Assertion and Perspective-is a superior metric for predicting decision-making process, as compared to raw counts of signature movements representing Assertion and Perspective (Connors et al., 2014). The current work linking MPA to observable decision-making process converges with recent studies, reviewed above, on individual differences in decision making, in two regards. First, there is recognition of the need for individuals to strike their own balance between complementary decision-making strategies. Second, these 
individually-defined propensities impact recordable behaviors during decision-making as studied in the laboratory.

The present study extends this work by delving deeper into the underlying elements of the Overall Factors of Assertion and Perspective in the MPA model, so as to examine if specific stages of decision-making motivation are especially predictive of individual differences in decision-making behavior. MPA theory posits, and assesses, three distinct stages in the decision-making process that require balancing the motivations (referred to as Action Motivations) underlying Assertion and Perspective (see Lamb, 2012; Moore, 2005). A first stage, Attention, involves the requisite process of scanning, probing, and deciphering all relevant information (Investigation, which is reflective of Assertion), along with surveying the broader scope represented by potential information (Exploring, which is reflective of Perspective). A second stage, Intention, is devoted to building the resolve necessary to move toward a decision (Determining, which is reflective of Assertion) while weighing the relative importance of information in hand as it relates to potential actions (Evaluating, which is reflective of Perspective). A final stage, Commitment, focuses on modulating the pace of implementing a decision (Timing, which is reflective of Assertion) while orchestrating the overall progression toward a decision and implications of the decision (Anticipating, which is reflective of Perspective). MPA records the unique individual patterning of Action Motivations across each of these stages, as individuals allocate their own balance between Assertion and Perspective differentially across all three stages.

In this study, we build on our prior experimental work showing how individually-defined balance between Assertion and Perspective predicts decision-making process in the laboratory to examine if these indicators are more telling at specific, consecutive motivational stages. We employed hypothetical decision-making scenarios. These have been widely used in both cognitive and political science and have been shown to have strong linkages to real-world decision making (Parker \& Fischhoff, 2005)—which permitted subjects the freedom to control their own information search via the option of making requests for more information prior to coming to a decision, with no specific time constraints.

We recruited a group of seasoned decision-makers - senior military officers with decades of experience - as notable differences in decision-making style such as initiation and maintaining intention have been observed in prior studies of military leadership (e.g., Thunholm, 2004), with implications for real-world behavior (Mintz, Redd, \& Vedlitz, 2006). Building on our prior focus on Assertion and Perspective (Connors et al., 2013; Connors et al., 2014), here we examine the extent to which the balance between Assertion and Perspective at each of the three successive stages in the MPA model predicts future decision-making process in the laboratory tasks.

\section{Methods}

\subsection{Subjects}

Twelve current or retired U.S. military officers participated in the study. They had between twenty and thirty years of military service, and represented all branches of the armed forces with the exception of the Army. There were three females and nine males. All subjects provided informed consent via a protocol approved by the appropriate Institutional Review Board.

\section{$2.2 M P A$}

Following the methodology of MPA, each subject participated in a two-hour interview with one MPA interviewer. During the interview, subjects were presented with a series of open-ended questions that focus on personal milestones, career history, and present situation. Posture-Gesture Mergers (PGMs) (as defined in the Introduction) were recorded by the interviewer, as expressed across multiple stages of the decision-making process (see Lamb, 2012; Moore, 2005). The PGMs are coded as representing one of six Action Motivation behaviors, with the three stages of decision making (Attention, Intention, Commitment) each comprising two Action Motivations, with one reflecting Assertion and another Perspective. The proportions of their total PGMs are then tallied across the six Action Motivations, with the distribution of proportions reflecting each individual's decision-making motivation. Connors et al. (2013) provide examples of such PGMs that would correspond to Assertion and to Perspective at each stage of the motivational decision-making process (Attention, Intention, Commitment).

Individual differences come into play as individuals find their own balance between the complementary factors of Assertion and Perspective. In our prior work we created a Perspective/Assertion Balance score-P/A Balance - which we define as follows: \% Perspective- $\%$ Assertion (Connors et al., 2013). With this difference score, a positive number reflects more distribution to Perspective, and a negative number reflects more distribution to Assertion. We have shown that this metric is coded with excellent reliability across raters and that 
it is more predictive of decision-making process, as assessed via completion of hypothetical decision-making scenarios, than raw counts of signature movements corresponding to the Overall Factors (Connors et al., 2014).

In this study, we applied the same approach to create difference scores, reflecting a balance between Assertion and Perspective, at each of the three stages of decision-making motivation, namely Attention, Intention, and Commitment. This metric provides a window into how each individual balances the need for both Assertion and Perspective at the more fine-grained level of staging throughout decision-making, permitting an opportunity to see if there are stages that are especially illuminating with respect to individual differences. The variables were created as follows:

\section{Attention Balance $=\%$ Exploring- $\%$ Investigating \\ Intention Balance $=\%$ Evaluating- $\%$ Determining \\ Commitment Balance $=\%$ Anticipating $-\%$ Timing}

In each case, a positive score indicates higher Perspective relative to Assertion, a negative score indicates higher Assertion relative to Perspective, and a 0 score indicates an even balance, specific to each of the three decision-making stages in the MPA model.

\subsection{Hypothetical Decision-Making Scenarios}

Four hypothetical decision-making tasks (Financial, Health, Voting, and Strategy) were designed for this study, drawing on prior use of this general paradigm in political science and other behavioral research (e.g., Dawes, 2007; Gartner, 2008; Mintz et al., 2006). Subjects completed each of these in a laboratory setting. During each task, subjects were given the option to seek out - one at a time-additional information to consider before coming to a decision, or to move on to make a final decision (see Connors et al., 2013, for an example of one scenario).

The number of information draws (requests for additional information) and response time (measured in seconds) were recorded electronically for each scenario. Information search and response time are presumed to be sensitive quantitative indicators of decision-making process that would reveal differences across individuals. To capture the process of decision-making - and not the actual decisions being made - we created two summary outcome measures based on subject behavior across all four scenarios. Total Response Time was computed as total chronological time (in seconds) summed across all four hypothetical scenarios. Total Info Draws was computed as the total number of requests for additional information summed across all four hypothetical scenarios. While noting a significant correlation between these outcome measures $(\mathrm{r}=.54, \mathrm{p}<.05)$, we consider both variables separately in our prediction models as we have done in prior work (Connors et al., 2013).

\subsection{Analytic Plan}

Given the exploratory nature of this investigation, we opted to perform multiple regression analysis with backward elimination (see Dunkler, Plishke, Leffondre, \& Heinze, 2014) for each of the outcome variables (Total Info Draws, Total Response Time), using the three Action Motivation balance scores as predictors. With a small data set and no a priori prediction, backward elimination provides a rational first step for determining a best-fitting model. It is noted that, by convention, the present sample size is small relative to the number of predictors for multiple regression. However, as this paper represents an initial exploration, we suggest that the fundamental issue comes down to having a sufficient number of subjects to detect the expected effect size in the model. Based on our prior work (Connors et al., 2013, 2014), we expect moderate to large effect sizes. As such, we favor an exploratory application of backward elimination, with the understanding that future work with larger samples would be needed to generate more stable and generalizable parameter estimates.

\section{Results}

Notable individual differences were found for all measures used in this study. Table 1 presents descriptive statistics on the three Action Motivation balance scores. The skew toward Assertion (as evidenced by mean scores which are negative) is consistent with the overall distributional pull toward Assertion in this sample (Connor et al., 2013). As discussed in prior publications (e.g., Connors et al., 2013), the Total Info Draws ranged from 10 to 21, and Total Response Time ranged from 365.62 seconds to 943.53 seconds. For each of these measures, there was a relatively equal distribution of scores between the two endpoints noted above. Notable covariation amongst the 3 Action Motivation balance scores as well as divergence was found. Attention Balance was distinct, correlating -.02 (ns) with Intention Balance, and .13 (ns) with Commitment Balance. Intention Balance and Commitment Balance were highly associated, correlating .80 (p .01), but were still considered as 
separable predictors in the backward elimination multiple regression models, which would factor in the observed collinearity.

Table 1. Descriptive statistics for the action motivation balance scores

\begin{tabular}{llll}
\hline Action Motivation Balance Scores & Minimum & Maximum & M (SD) \\
\hline Attention Balance & -24.00 & 6.00 & $-10.50(7.50)$ \\
Intention Balance & -21.00 & 10.00 & $-6.58(9.40)$ \\
Commitment Balance & -20.00 & 20.00 & $-5.58(12.68)$ \\
\hline
\end{tabular}

Note. $\mathrm{M}=$ mean, $\mathrm{SD}=$ standard deviation

For each of the outcome measures, we ran a backward elimination multiple regression model, which initiated with all 3 Action Motivation balance scores, and then employed a probability of F-to-remove of $>=.100$ in sequence. Table 2 presents the results of the 3 iterations that emerged for Total Response Time, and Table 3 presents the results of the 3 iterations that emerged for Total Info Draws.

Table 2. Backward elimination multiple regression model predicting total response time from action motivation balance scores

\begin{tabular}{llll}
\hline Model & \multicolumn{2}{l}{ Unstandardized Coefficients } & \multicolumn{2}{l}{ Standardized Coefficients } \\
\hline & $\mathrm{B}$ (Std. Error) & Beta & $\mathrm{t}(\mathrm{p})$ \\
\cline { 2 - 4 } 1: & $0.018(0.153)$ & .037 & $.119(.908)$ \\
Attention Balance & $0.242(0.141)$ & .618 & $1.715(.125)$ \\
Intention Balance & $-0.023(0.183)$ & -.047 & $-.123(.905)$ \\
Commitment Balance & & & \\
2: & $0.237(.128)$ & .606 & $1.85(.097)$ \\
Intention Balance & $-0.014(0.157)$ & -.028 & $-.087(.933)$ \\
Commitment Balance & & & \\
3: & $17.603(1.114)$ & .590 & $2.311(.043)$ \\
Intention Balance & &
\end{tabular}

Note. $\mathrm{B}=$ unstandardized beta coefficient, Std. Error $=$ standard error, $\mathrm{t}=\mathrm{t}$ statistic, $\mathrm{p}=$ probability associated with $\mathrm{t}$ value.

Table 3. Backward elimination multiple regression model predicting total info draws from action motivation balance scores

\begin{tabular}{llll}
\hline Model & \multicolumn{2}{l}{ Unstandardized Coefficients } & \multicolumn{2}{l}{ Standardized Coefficients } \\
\hline \multirow{2}{*}{$\begin{array}{l}\text { B (Std. Error) } \\
\text { Attention Balance }\end{array}$} & $0.726(7.696)$ & Beta & $\mathrm{t}(\mathrm{p})$ \\
\cline { 2 - 4 } Intention Balance & $4.366(7.077)$ & .028 & $.094(.927)$ \\
Commitment Balance & $-3.145(9.180)$ & .700 & $2.030(.077)$ \\
2: & & -.125 & $-.343(.741)$ \\
Intention Balance & $14.189(6.437)$ & & \\
Commitment Balance & $-2.792(7.906)$ & .691 & $2.20(.055)$ \\
3: & & -.111 & $-.353(.732)$ \\
Intention Balance & $12.892(5.049)$ & & .628 \\
\hline
\end{tabular}

Note. $\mathrm{B}=$ unstandardized beta coefficient, $\mathrm{Std}$. Error=standard error, $\mathrm{t}=\mathrm{t}$ statistic, $\mathrm{p}=$ probability associated with $\mathrm{t}$ value. 
The results converged on the same best-fit model for both outcome predictors. Intention Balance emerged as a significant predictor of both Total Response Time and Total Info Draws, whereas Attention Balance and Commitment Balance were excluded based on the statistical model employed. Thus this staging in the MPA model seems to be an especially salient predictor of decision-making process as elicited using the particular paradigm employed in this study. It is notable that the magnitude of the effect was substantial as well, as reflected in the Beta estimates shown in Tables 2 and 3. The positive nature of the Beta estimates suggests that the degree to which an individual is driven by a motivation toward Perspective is predictive of requesting more information draws, and having a longer response time, when involved in the decision-making scenarios. Furthermore, it is informative to reference the variance explained in each final model. For Total Response Time, the unadjusted $\mathrm{R}^{2}$ was $35 \%$, and the adjusted $\mathrm{R}^{2}$ was $28 \%$. For Total Info Draws, the $\mathrm{R}^{2}$ was $40 \%$, and the adjusted $\mathrm{R}^{2}$ was $28 \%$. These values suggest a strong predictive value of MPA in general on decision-making process, and the specific potential offered via assessment of Intention.

\section{Discussion}

The results of this study build on our prior papers (Connors et al., 2013; Connors et al., 2014) and add empirical evidence that signature movement patterns, as revealed by use of MPA, yield important signals on decision-making style and are predictive of decision-making process. While a number of laboratory-based protocols have been used to elicit individual differences in decision making (as reviewed in our Introduction), there have been few attempts to generate methods that offer some prognostic value, particularly going beyond the inherent limitations of self-report (Connors et al., 2013). Thus our accumulating data supporting the utility of MPA as a predictor of notable individual differences in leaders speaks to the potential that comes from applying observational methodologies in general, and specially those that are movement-based, to reveal reliable indicators of decision-making style that are predictive of future decision-making process.

The specific contribution here is to plumb the motivational stages of decision-making posited in the MPA model (Lamb, 2012; Moore, 2005). Our primary finding is that Intention, the middle of the 3 stages in MPA, was the most salient motivational indicator of observed individual differences in decision-making process. As noted earlier, the MPA model suggests that Intention is devoted to Determining (building the resolve necessary to move toward a decision) while also Evaluating (weighing the relative importance of information in hand as it relates to potential actions). Determining is one of the Action Motivations that comprise Assertion, while Evaluating is one of the processes underlying Perspective. Our findings suggest that the way an individual balances, in terms of complementary underlying motivations associated with Intention, the need for determination when formulating a decision to push and get results while prioritizing the available information to design results is an especially sensitive indicator of decision-making style. An inclination toward Evaluating leads individuals, in the protocol used in this study, to reach out for more information before coming to a decision (as indexed by total info draws), and to spend more time arriving at a decision (as indexed by total response time). It is noted that information draws and response time are, in this study, proximal indicators of an overall decision-making process, and the linkages with MPA suggest that signature movement patterns can provide insight into a general propensity to weigh the need for action (Assertion) versus the need for reflection (Perspective), and that such individual differences are most telling during the Intention stage.

It is critical to emphasize that all subjects need to employ both Determining coded relative to what the subject's body is revealing through increasing or decreasing pressure, and Evaluating coded in how the body shapes to rise and descend along the vertical dimension to size up an issue from above and below. What this study demonstrates is the predictive potential of deciphering individuals' motivational tendencies as referenced to stages of decision making, which converge with observable behavioral differences when faced with decision-making tasks. In this sense, this work converges with a theme emerging from a number of disciplines and methodologies used to uncover individual differences in decision making - there are a multitude of processes that need to be evoked, and individual differences arise in the differing priorities individuals give when balancing complementary cognitive strategies.

We do not claim that the other stages of decision-making motivation in the MPA model are irrelevant. In the MPA model, the Intention stage is a bridge between Attention (which involves a focus on information) and Commitment (in which a decision is implemented). Rather, we say that Intention, rather than Attention or Commitment, emerged as the best predictor of individual differences in the specific aspects of decision-making process required in the experimental paradigm used in this study. Variation in the motivational balance applied to Perspective and Assertion in Attention and Commitment is revealed to predict other aspects of decision-making process in future studies. To that point, we again emphasize the complexity of cognitive and motivational processes that underlie decision-making in general, and the multitude of indicators that may reveal 
multiple bases of individual differences in process and style when faced with a range of decision-making tasks. Furthermore, our work has been devoted to military leaders and it may be that Intention is a particularly relevant stage for this population that drives differences in decision-making style. While future work will be necessary to build on the findings reported in this paper, the results do add credence to the suggestion that movement-based methods such as MPA offer potentially powerful approaches for gaining insight into the motivational bases of decision-making style and may offer prediction of future decision-making behavior in leaders of interest, particularly as movement can be observed from videotape as well as in person.

In conclusion, we note considerations of the study design we employed. In addition to focusing on one specific type of leader, our sample was modest in size, in part due to the intensive nature of conducting both MPA and the experimental protocol. The results of the regression analyses, while sensitive to the effect sizes observed in our sample using these methodologies, would need to be replicated with larger samples in order to provide more stable estimations of parameters in the models. Future research that broadens this focus would be welcome. In addition, additional types of decision-making process may be studied experimentally and would offer fertile ground for expanding our understanding of the potential utility of MPA as a predictor of decision-making process. Overall, this work adds to the growing emphasis on bringing attention to the notable individual differences in decision-making process that may be found in the general population, and specifically in various types of leaders who are charged with making complex decisions on a regular basis.

\section{Acknowledgments}

We wish to thank the subjects who participated in this study for their time and effort. We wish to thank the Movement Pattern Analysis (MPA) gold standard coders for their support of the research coding process.

\section{References}

Barnes, K. A., Anderson, K. M., Plitt, M., \& Martin, A. (2014). Individual differences in intrinsic brain connectivity predict decision strategy. Journal of Neurophysiology, 112, 1838-1848. http://dx.doi.org/ 10.1152/jn.00909.2013

Carnevale, J. J., Inbar, Y., \& Lerner, J. S. (2011). Individual differences in need for cognition and decision-making competence among leaders. Personality and Individual Differences, 51, 274-278. http://dx.doi.org/10.1016/j.paid.2010.07.002

Connors, B. L. (2006). No leader is ever off stage: Behavioral analysis of leadership. Joint Force Quarterly, 43, 83-87.

Connors, B. L., Rende, R., \& Colton, T. J. (2013). Predicting individual differences in decision-making process from signature movement styles: An illustrative study of leaders. Frontiers in Psychology, 4, Article 658,

Connors, B. L., Rende, R., \& Colton, T. J. (2014). Inter-rater reliability for movement pattern analysis (MPA): Measuring patterning of behaviors versus discrete behavior counts as indicators of decision-making style. Frontiers in Psychology, 5, Article 605. http://dx.doi.org/10.3389/fpsyg.2014.00605

Dawes, C. T., Fowler, J. H., Johnson, T., McElreath, R., \& Smirnov, O. (2007). Egalitarian Motives in Humans. Nature, 446, 794-796. http://dx.doi.org/10.1038/nature05651

Doll, B. B., Shohamy, D., \& Daw, N. D. (2015). Multiple memory systems as substrates for multiple decision systems. Neurobiology of Learning and Memory, 117, 4-13. http://dx.doi.org/10.1016/ j.nlm.2014.04.014

Dunkler, D., Plischke, M., Leffondre, K., \& Heinze, G. (2014). Augmented backward elimination: A pragmatic and purposeful way to develop statistical models. PLoS ONE, 9(11), e113677.

Eppinger, B., Walter, M., Heekeren, H. R., \& Li, S. C. (2013). Of goals and habits: Age-related and individual differences in goal-directed decision-making. Frontiers in Neuroscience, 7, Article 253. http://dx.doi.org/ 10.3389/fnins.2013.00253

Gartner, S. S. (2008). The multiple effects of casualties on public support for war: An experimental approach. American Political Science Review, 102, 95-106. http://dx.doi.org/10.1017/S0003055408080027

Hansen, K. A., Hillenbrand, S. F., \& Ungerleider, L. G. (2012). Human brain activity predicts individual differences in prior knowledge use during decisions. Journal of Cognitive Neuroscience, 24, 1462-1475. http://dx.doi.org/10.1162/jocn_a_00224

Lamb, W. (2012). A Framework For Understanding Movement: My Seven Creative Concepts. London: Brechin Books. 
Laureiro-Martinez, D. et al. (2014). Frotopolar cortex and decision-making efficiency: Comparing brain activity of experts with different professional background during an exploration-exploitation task. Frontiers in Human Neuroscience, 7, Article 927. http://dx.doi.org/10.3389/fnhum.2013.00927

Mintz, A., Redd, S. B., \& Vedlitz, A. (2006). Can we generalize from student experiments to the real world in political science, military affairs, and international relations? Journal of Conflict Resolution, 50, 757-776. http://dx.doi.org/10.1177/0022002706291052

Mishra, S. (2014). Decision-making under risk: Integrating perspectives from biology, economics, and psychology. Personality and Social Psychology Review, 18, 280-307. http://dx.doi.org/10.1177/ 1088868314530517

Moore, C. L. (2005). Movement and Making Decisions: The Body-Mind Connection in the Workplace. New York: Rosen Publishing Group.

Parker, A. M., \& Fischhoff, B. (2005). Decision-making competence: External validation through an individual-differences approach. Journal of Behavioral Decision Making, 18, 1-27. http://dx.doi.org/ 10.1002/bdm.481

Perri, R. L., Berchicci, M., Spinelli, D., \& Di Russo, F. (2014). Individual differences in speed and accuracy are associated to specific brain activities of two interacting systems. Frontiers in Behavioral Neuroscience, 8 , Article 251. http://dx.doi.org/10.3389/fnbeh.2014.00251

Thunholm, P. (2004). Decision making style: Habit, style, or both? Personality and Individual Differences, 36, 931-944. http://dx.doi.org/10.1016/S0191-8869(03)00162-4

White, C. N. et al. (2014). Decomposing decision components in the stop-signal task: A model-based approach to individual differences in inhibitory control. Journal of Cognitive Neuroscience, 26, 1601-1614. http://dx.doi.org/10.1162/jocn_a_00567

\section{Copyrights}

Copyright for this article is retained by the author(s), with first publication rights granted to the journal.

"The views expressed in this article are those of the authors and do not reflect the official policy or position of the Department of Defense or the US Government." 